\title{
Production technology of solid-sealed polar pole
}

\author{
Li Dongsheng , Sun Zhonghang , Li Xupei \\ Pinggao Group Ltd. Pingdingshan Henan China \\ 15238237753@163.com,13525391231@163.com,15638691127@163.com
}

Keywords: solid-sealed polar pole; epoxy; APG; technology

Abstract: Production technology of $12 \mathrm{kv}$ solid-sealed polar pole is mainly expounded, including mould installation, spares preparation, mix preparation, injection molding, after treatment, assembling, inspection, etc., with focus on spare preparation, mix preparation and injection molding.

\section{Introduction}

The vacuum breaker dominates the domain of medium voltage, especially $12 \mathrm{kV}$ voltage level. The polar pole insulation of vacuum breaker has gone through air insulation-compound insulation-solid-sealed insulation. The third generation insulation, i.e. solid-sealed polar pole, is an integrated polar pole formed by solidifying and integrating vacuum quenching pot, conductive terminal, etc. with epoxy resin through APG technology. As vacuum quenching pot is enveloped in epoxy, it is hardly affected by external contamination and moisture, among other adverse effects. Besides, epoxy does not only serve as major insulation of quenching pot, but also is mechanical support of quenching pot, with electric field distribution outperforming traditional assembled polar pole, decreased structural components, enhanced reliability of galvanic circle, hence its increasingly wider application.

\section{Overview of $12 \mathrm{kV}$ solid-sealed polar pole production technology}

Solid-sealed polar pole is mainly composed of vacuum quenching pot, upper outlet, lower outlet, radiating fin, insulation pull rod, fixing inset, epoxy layer. The flowchart of production technology of solid-sealed polar pole is as shown in figure 1 below.

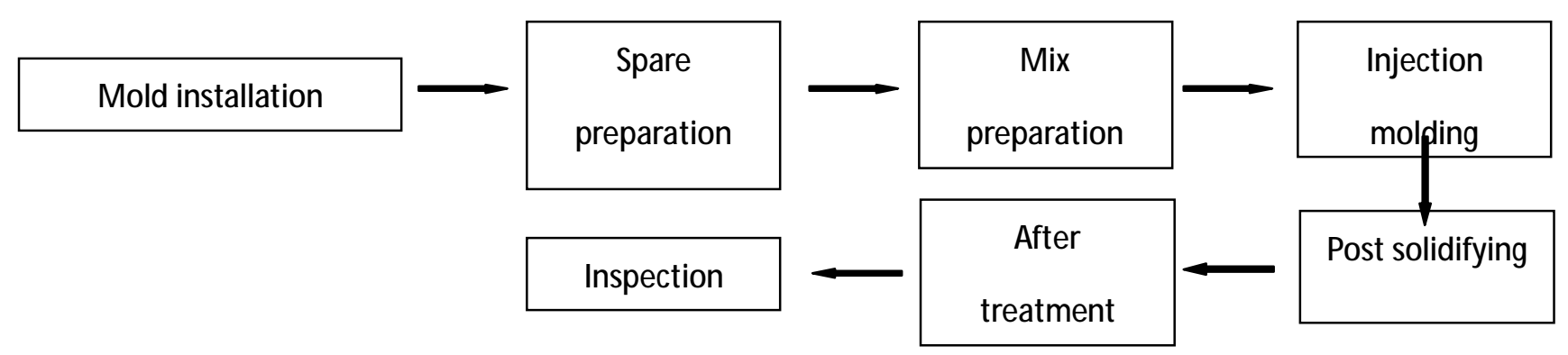

Figure 1 Flowchart of production technology of solid-sealed polar pole 


\section{Mould installation}

Mold is essential to the quality of solid-sealed polar pole produced by APG technology, especially the appearance quality. The mold suitable for epoxy APG technology is required to have high cavity airtightness, high smooth finish of mold cavity, convenience in demolding, feed inlet located on lower part of mold, and exhaust trough left on upper part of mold.

Installation sequence of mold is usually lower loose core, static mold, moving mold, upper loose core. Keep location pin of static mold and moving mold and locating pin of upper and lower loose core under observation when installing mold to ensure locating pin is located at center of locating hole. Close static and moving mold as well as upper and lower loose core and pressurize to dwelling state, use feeler gauge to check assembly seam of mold to prevent large seal to cause leakage of materials.

\section{Spare preparation}

Cleanse the parts to be embedded in epoxy such as vacuum quenching pot, upper outlet, lower outlet, insert, etc. Cleanse using alcohol before casting to remove the oil pollution and dust on surface of spares.

Monopole assembly. Monopole assembly is assembly of vacuum quenching pot and spares such as upper and lower outlet. In APG casting, monopole assembly is to be installed in precise APG mold, which puts forward higher requirements on precision of monopole assembling, requiring end face of upper and lower outlet to be parallel, the center distance of upper and lower outlets to have small error, so assembling process of monopole assembly must be carried out on locating tooling.

Spare warm-up. While warming up the mold, put insert and monopole assembly in oven for warm-up for about 2 hours, make the warm-up temperature of monopole assembly and insert approximate the mold temperature.

Coat adhesive agent to surface of vacuum quenching pot. To guarantee tight coupling of the silicagel layer on surface of vacuum quenching pot with epoxy in casting, it is required to evenly smear a layer of adhesive agent on the silicagel layer on surface of vacuum quenching pot.

\section{Mix preparation}

APG technology puts forward higher requirements on epoxy mix than vacuum casting material, i.e. stableness at room temperature, high reaction activity at high temperature, capacity of rapid gelation, short solidification cycle, good resistance to cracking and impact, high resistance to mechanical and electrical stress [1]. The solid-sealed polar pole has complicated interior mechanism and large insert, hence its high requirements on material's resistance to cracking. Due to the influence of uncertainties such as external environment in the forming process of product, the material is required to have good processing property and wider scope of application to reduce influence of uncertainties such as temperature, humidity, vibration, etc. See table 1 for performance indices APG casting epoxy material system applicable to solid-sealed polar pole.

Table 1 Performance indices for epoxy material system for APG use

\begin{tabular}{|l|c|c|}
\hline Performance index & Scope & Inspecting standard \\
\hline Tensile strength,N/mm2 & $70 \sim 90$ & \multirow{2}{*}{ GB/T 2567-2008 } \\
\hline Bending strength, $\mathrm{MPa}$ & $110 \sim 130$ & GB/T 12007.7-1989 \\
\hline Impact strength, $\mathrm{kJ} / \mathrm{m} 2$ & $9 \sim 11$ & GB/T 11998-1989 \\
\hline Gelation time $\left(130^{\circ} \mathrm{C}\right) / \mathrm{min}$ & $10 \sim 30$ & GB/T 1034-2008 \\
\hline Glass transition temperature, ${ }^{\circ} \mathrm{C}$ & $100 \sim 130$ & $0.1 \sim 0.2$ \\
\hline $\begin{array}{l}\text { Hydroscopicity, weight change } \\
60 \mathrm{~min} / 100^{\circ} \mathrm{C}\end{array}$ & & \\
\hline
\end{tabular}


Before APG casting, epoxy mix should be formulated by blending epoxy, hardener, filling and toner even and degasifying thoroughly. Whip and vacuumize mix using whipping and mixing device for about 2 hours. The vacuum degree is below $200 \mathrm{~Pa}$.

\section{Injection molding}

APG technology is automatic pressure gelation, mainly applied to produce medium and high voltage epoxy insulation products, characterized in that it can shorten the production cycle of epoxy insulation products from a dozen of hours in traditional vacuum casting to a dozen of minutes and better control exothermic effect, conductive to compensating for contraction caused by solidifying reaction, plus wonderful dimensional stability and high mechanical strength, etc. With structural features of complicated shape, thin wall and multi-azimuth loose core, solid-sealed polar pole is more suitable to be produced by APG technology [2].

See table 2 for APG casting technology parameters suitable for solid-sealed polar pole.

Table 2 APG casting technology parameters

\begin{tabular}{|c|c|c|}
\hline $\begin{array}{c}\text { Processing } \\
\text { parameter }\end{array}$ & Unit & Requirement \\
\hline temperature & ${ }^{\circ} \mathrm{C}$ & $130-160$ \\
\hline $\begin{array}{c}\text { Injection } \\
\text { pressure }\end{array}$ & $\mathrm{MPa}$ & $0.1-0.2$ \\
\hline Feeding time & $\mathrm{min}$ & $5-15$ \\
\hline Dwell pressure & $\mathrm{MPa}$ & $0.4-0.8$ \\
\hline Dwell time & $\mathrm{min}$ & $20-40$ \\
\hline
\end{tabular}

For the demolded solid-sealed polar pole, the work of dismantling mold accessory, repairing and cleaning, etc, should be done. Lest just demolded solid-sealed polar pole should overstand at room temperature, solid-sealed polar pole should be put in solidifying over as quickly as possible for post-solidifying.

\section{After treatment}

Grind feed sprue and to-be-repaired bubble hole position, the feed sprue and to-be-repaired bubble hole position of grinded product should be leveled. Pay attention to not to harm the position near feed sprue when grinding.

Surface treatment of product: first wipe off the sundries and dust, etc. on surface with cloth, then wipe grinded area using cloth moistened with brightener.

Assembly of insulation pull rod and radiating fin. Reference recommended torque of manufacturer of vacuum quenching pot for tightening torque of insulation pull rod, generally being $35-60 \mathrm{~N} \cdot \mathrm{m}$. In assembling radiating fin, tightening torque is generally $20-35 \mathrm{~N} \cdot \mathrm{m}$ according to bolt size.

\section{Inspection}

Visual inspection. The surface should be smooth and leveled without burr, pores, cracks, sharp corners, mechanical damage, among other defects.

Size should conform to requirements in drawing.

Screw hole inspection: check whether screw holes in upper and lower outlets and bottom inserts can enable the bolt to be screwed down smoothly. If not, improve the screw hole.

Check interior for pores, impurities and cracks using defectoscope

Power frequency pressure test is qualified.

Self discharge test, not greater than $5 \mathrm{pC}$.

Closing force and counterforce passes examination.

Loop resistance passes examination. 


\section{Reference:}

[1]Xu Xiangqian. Popularization and Application of Automatic Pressure Gelation Process (APG) [J] Huatong Technology,1998 4),P.19 - 22,27.

[2]Pang Mingwei State of the Art of APG and Technological Equipment [C]// National Epoxy Casting and APG Engineering Workshop,2004,P.15 - 31 